\title{
Efeito protetor do bentazon sobre a fitotoxicidade de herbicidas inibidores de ALS em duas cultivares de feijoeiro
}

\author{
Safening effect of bentazon over phytotoxicity of ALS inhibitors herbicides on two cultivars of \\ common beans
}

\author{
Lucas De Ross Marchioretto ${ }^{1 \star} \&$ Taísa Dal Magro² \\ ${ }^{1}$ Universidade do Estado de Santa Catarina, Lages, SC, Brasil. Autor para correspondência: lucasdeross@hotmail.com. \\ ${ }^{2}$ Universidade de Caxias do Sul, Caxias do Sul, RS, Brasil.
}

\begin{abstract}
RESUMO
O presente experimento teve por objetivo avaliar a seletividade de herbicidas e o controle de plantas daninhas, na pós-emergência da cultura do feijoeiro. Foram testados os herbicidas cloransulam e imazethapyr, isoladamente e em associação com bentazon e comparados com uma testemunha capinada e uma infestada, nas cultivares de feijão Agronorte (Anfc9) e IPR Uirapuru. O experimento foi conduzido a campo, em delineamento de blocos casualizados, com quatro repetições. Foram analisadas: fitotoxidez, controle de plantas daninhas, altura de inserção de primeiro legume, número de legumes por planta, peso de mil grãos e produtividade. Cada cultivar possui nível de tolerância diferente aos herbicidas cloransulam e imazethapyr. A cultivar ANfc9 foi, em geral, mais tolerante aos herbicidas que a IPR Uirapuru. A adição de bentazon aos herbicidas cloransulam ou imazethapyr, diminui a fitotoxidez causada à cultura do feijão, cultivares Agronorte (Anfc9) e IPR Uirapuru e não altera o controle de plantas daninhas, demonstrando não haver antagonismo na mistura.
\end{abstract}

PALAVRAS-CHAVE: cloransulam, imazethapyr, fitotoxidez, tolerância varietal, Phaseolus vulgaris.

\begin{abstract}
The experiment aimed to test the crop selectivity and postemergence weed control on common beans. The treatments consisted of cloransulam, and imazethapyr isolated and tank-mixed with bentazon, a weedy control and a weed-free control for comparison of the cultivars Agronorte (ANfc9) and IPR Uirapuru of common beans. The experiment was conducted on a field, on a complete randomized block experimental design with four replications. The variables analyzed were crop injury, weed control, first pod height, pod number, 1000 grains weight and crop yield. Each cultivar behaves differently with the ALS herbicides. The cultivar ANfc9 was more tolerant to the ALS herbicides than IPR Uirapuru. Bentazon tankmix with cloransulam or imazethapyr decreased the level of crop injury and no antagonism was observed to weed control.
\end{abstract}

KEYWORDS: cloransulam, imazethapyr, crop injury, varietal tolerance, Phaseolus vulgaris.

\section{INTRODUÇÃO}

O feijão é um dos alimentos mais importantes no Brasil, por ser muito nutritivo e ter preço acessível para as classes menos favorecidas. Como consequência, a produção de feijão é bastante expressiva no Brasil, pois há uma alta demanda no mercado consumidor interno. Na safra de 2015/16, o Brasil produziu 3.334,6 mil toneladas de grãos (CONAB 2016).

Dentre os entraves que os produtores rurais se deparam quando cultivam o feijoeiro, a necessidade de controle de plantas daninhas é um dos principais problemas, pois as mesmas possuem rápido crescimento em relação à cultura agrícola, competindo por água e nutrientes além de dificultar a colheita mecanizada, causando redução da produtividade. As principais plantas daninhas magnoliopsidas encontradas nos sistemas de cultivo na região dos Campos de Cima da Serra são picão-preto (Bidens pilosa), nabo (Raphanus spp.), buva (Conyza spp.), corda-de-viola (Ipomoea spp.) e leiteira (Euphorbia heterophylla). 
Na cultura do feijão, as plantas daninhas afetam a produtividade da cultura no período compreendido entre os estádios V4 e R6, cuja área deve estar totalmente livre de plantas daninhas (KOZLOWSKI et al. 2002). Os herbicidas seletivos, de forma geral, devem controlar as plantas daninhas e não causar fitotoxidez que possa reduzir a produtividade da cultura. O controle químico consiste no uso de substâncias que uma vez absorvidos pelas plantas daninhas, afetam seu metabolismo e podem matá-la ou reduzir seu crescimento (CONSTANTIN 2011).

No Brasil, o herbicida imazethapyr é registrado para uso em pós-emergência na cultura do feijão no controle de plantas daninhas mono e dicotiledôneas (AGROFIT 2017) e o herbicida cloransulam é reportado em experimentos realizados em outros países como seguro para uso pós-emergente da cultura do feijão (SOLTANI et al. 2013) desde que em doses menores daquela recomendada para a cultura da soja no Brasil (40 g i.a. ha-1). Esses autores encontraram baixo nível de fitotoxidez visual para a cultura apesar de moderada redução na produtividade de grãos. A adição de bentazon ao cloransulam provocou diminuição severa dos efeitos fitotóxicos provocados pelo cloransulam (SOLTANI et al. 2012) e imazethapyr (BAUER et al. 1995b).

No Brasil, pouco se sabe sobre os efeitos de sinergismo ou antagonismo sobre a seletividade e controle de plantas daninhas na cultura do feijoeiro promovidos pela associação de herbicidas inibidores de ALS com bentazon. Desta maneira, o objetivo deste experimento foi avaliar o controle de plantas daninhas e a seletividade dos herbicidas aplicados em pós-emergência para as cultivares de feijão Agronorte (Anfc9) e IPR Uirapuru.

\section{MATERIAL E MÉTODOS}

A pesquisa foi conduzida a campo na Fepagro Nordeste localizada na BR 285, km 5 no município de Vacaria, RS, no ciclo produtivo de 2014/2015. A área experimental utilizada estava a três anos em pousio e as plantas daninhas não foram controladas durante esse período. O experimento foi conduzido em delineamento experimental de blocos ao acaso, em esquema fatorial com sete tratamentos e duas cultivares de feijão, em quatro repetições. Cada parcela foi composta de $2 \mathrm{~m}$ de largura e $5 \mathrm{~m}$ de comprimento, totalizando área de $10 \mathrm{~m}^{2}$; como área útil foram descartadas $0,5 \mathrm{~m}$ (bordas), totalizando área de $4 \mathrm{~m}^{2}$ ( $1 \mathrm{~m}$ de largura $\times 4 \mathrm{~m}$ de comprimento). As linhas de feijão foram semeadas em espaçamento de $0,50 \mathrm{~m}$ e foi utilizado as cultivares de feijão carioca Agronorte ANfc9 e IPR Uirapuru.

Os tratamentos consistiram de: Basagran $600^{\circledR}$ - Bentazon (720 g i.a. ha-1), Pacto ${ }^{\circledR}$ - Cloransulam methyl (17,5 g i.a. ha-1), Vezir ${ }^{\circledR}$ - Imazethapyr (100 g i.a. ha- $\left.{ }^{-1}\right)$, Pacto ${ }^{\circledR}+$ Basagran $600^{\circledR}$ - Cloransulam-methyl + Bentazon $(17,5+720$ g i.a. ha-1 $)$, Vezir ${ }^{\circledR}+$ Basagran $600^{\circledR}$ - Imazethapyr + Bentazon $(100+720$ g i.a. $\left.\quad a^{-1}\right)$, Testemunha Capinada e Testemunha Infestada.

A aplicação dos tratamentos ocorreu quando o feijão encontrava-se no estádio vegetativo V2 e as plantas daninhas em estádios iniciais de desenvolvimento. A aplicação foi realizada utilizando-se pulverizador costal pressurizado com $\mathrm{CO}_{2}$, a pressão constante de $2,5 \mathrm{kgf} \mathrm{cm}^{-2}$, contendo barra de aplicação provida de bicos com pontas de pulverização do tipo leque XR 110.02 calibrada para proporcionar a aplicação de um volume de calda de $150 \mathrm{~L} \mathrm{ha}^{-1}$. As principais plantas daninhas presentes na área foram picão preto, losna e milhã.

O controle foi avaliado aos 14, 21 e 28 dias após a aplicação dos tratamentos (DAT), analisando-se visualmente em escala de zero a $100 \%$, tendo como referência a testemunha infestada, onde zero corresponde a nenhum controle e $100 \%$ ao controle total das plantas daninhas. Os sintomas de fitotoxidez foram avaliados aos 7, 14 e 21 DAT, analisando-se visualmente em escala percentual de zero a $100 \%$, tendo como a referência a testemunha capinada, onde zero corresponde a nenhum sintoma e $100 \%$ a morte total das plantas. Foram escolhidas aleatoriamente 20 plantas em cada parcela para medir a estatura de planta e inserção de primeiro legume. Após a colheita, 20 plantas foram selecionadas aleatoriamente para a contagem do número de legumes. Para calcular a produtividade, foram colhidas a área útil das parcelas e os pesos foram corrigidos para a umidade de $13 \%$ para a obtenção da média de produtividade expressa em $\mathrm{kg} \mathrm{ha}^{-1}$. Também foi calculado a peso de mil grãos, a partir da produção de cada parcela.

Buscando atender as pressuposições da ANOVA, os dados de fitotoxidez, controle e produtividade foram transformados para raíz quadrada $(\sqrt{x})$. Os dados foram submetidos à análise de variância $(p \leq 0,05)$ e os tratamentos foram avaliados por comparação múltipla de médias pelo teste de Tukey $(p \leq 0,05)$. Os dados da variável controle foram comparados pelo teste $\mathrm{F}$ com o controle estimado pelo modelo de COLBY (1967) para as interações entre os herbicidas, utilizando-se o software estatístico Winstat v.2.0. 


\section{RESULTADOS E DISCUSSÃO}

$\mathrm{Na}$ variável controle (Tabela 1), todos os latifolicidas controlaram picão preto, com exceção de imazethapyr isolado o qual diferiu da testemunha infestada, tanto na avaliação dos 21 como dos 28 DAT. Foi considerado como controle insatisfatório quando as plantas daninhas apresentaram sinais de recuperação após os 21 DAT. Aos 21 DAT, os herbicidas que controlaram losna branca foram cloransulam, de forma isolada ou associado com bentazon; já aos 28 DAT, além desses imazethapyr, associado com bentazon, não diferiram da testemunha capinada. As plantas de milhã foram controladas apenas pelos tratamentos contendo imazethapyr, de forma isolada ou em associação com bentazon, porém com índices inferiores a testemunha capinada. Não houve antagonismo no controle das espécies latifólias avaliadas neste experimento pela associação dos herbicidas cloransulam ou imazethapyr, associados ao bentazon, conforme indicado pelo modelo de COLBY (1967) (Tabela 1). Entretanto, com picão e milhã, houve um atraso no controle promovido pela associação entre imazethapyr com o bentazon, aos 21 DAT, sem prejuízo de controle aos 28 DAT. Estes resultados corroboram com os observados por SOLTANI et al. (2013) em que a associação de bentazon ao cloransulam, na dose de $17,5 \mathrm{~g}$ i.a. ha ${ }^{-1}$, não afetou o controle de plantas daninhas dicotiledôneas. O controle de plantas daninhas dicotiledôneas pode ser decrescido quando o imazethapyr está associado ao bentazon, especialmente em doses menores, assim aumentando-se a dose de imazethapyr o antagonismo pode ser minimizado (BAUER et al. 1995c). O antagonismo geralmente ocorre quando a espécie daninha é mais sensível ao imazethapyr do que ao bentazon, ou seja, uma planta daninha que seria eficazmente controlada por imazethapyr isolado do que bentazon, tem seu controle reduzido pela adição de bentazon. Quando a planta daninha é mais sensível ao bentazon do que ao imazethapyr, o antagonismo não é observado assim, o herbicida que é mais propenso a ser antagonizado é o imazethapyr, quando associado ao bentazon (CANTWELL et al. 1989).

Tabela 1. Controle (\%) de picão preto (Bidens pilosa), losna branca (Parthenium hysterophorus) e milhã (Digitaria spp.), em função dos tratamentos aplicados em pós-emergência, avaliados aos 21 e 28 dias após a aplicação dos tratamentos (DAT). Vacaria, RS, 2017.

Table 1. Control (\%) of Bidens pilosa, Parthenium hysterophorus and Digitaria spp. in function of the postemergence treatments evaluated at 21 and 28 days after treatments (DAT). Vacaria, RS, 2017.

\begin{tabular}{|c|c|c|c|c|c|c|c|c|c|c|c|c|}
\hline \multirow[t]{2}{*}{ Tratamentos } & \multicolumn{4}{|c|}{ Picão preto } & \multicolumn{4}{|c|}{ Losna branca } & \multicolumn{4}{|c|}{ Milhã } \\
\hline & $21 \mathrm{DAT}$ & Colby & 28 DAT & Colby & $21 \mathrm{DAT}$ & Colby & 28 DAT & Colby & 21 DAT & Colby & 28 DAT & Colby \\
\hline Bentazon $^{2}$ & $97 \mathrm{a}$ & - & $100 \mathrm{a}$ & - & $32 \mathrm{c}$ & - & $63 \mathrm{~b}$ & - & $0 \mathrm{~d}$ & - & $0 \mathrm{c}$ & - \\
\hline Bent.+cloran & $74 a b^{n s}$ & 100 & $88 a b^{n s}$ & 100 & $93 a b^{\text {ns }}$ & 85 & $99 \mathrm{a}^{\mathrm{ns}}$ & 99 & $0 \mathrm{~d}^{\mathrm{ns}}$ & 0 & $1 \mathrm{c}^{\mathrm{ns}}$ & 0 \\
\hline Bent. & $76 a b^{*}$ & 98 & $96 \mathrm{a}^{\mathrm{ns}}$ & 100 & $53 b c^{n s}$ & 43 & $80 a^{\text {ns }}$ & 89 & $41 c^{*}$ & 74 & $70 b^{n s}$ & 82 \\
\hline Clora & $91 \mathrm{a}$ & - & $96 \mathrm{a}$ & - & $85 a b$ & - & $98 \mathrm{a}$ & & $0 d$ & - & $7 \mathrm{c}$ & . \\
\hline maz & $45 \mathrm{~b}$ & - & $73 \mathrm{~b}$ & - & $38 \mathrm{c}$ & - & $70 \mathrm{~b}$ & - & $74 \mathrm{~b}$ & - & $83 \mathrm{~b}$ & - \\
\hline Test. & $100 \mathrm{a}$ & - & $100 \mathrm{a}$ & - & $100 \mathrm{a}$ & - & $100 \mathrm{a}$ & - & $100 a$ & - & $100 \mathrm{a}$ & 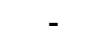 \\
\hline Test Infest & $0 \mathrm{c}$ & - & $0 \mathrm{c}$ & - & $0 \mathrm{~d}$ & - & $0 \mathrm{c}$ & - & $0 \mathrm{~d}$ & - & $0 \mathrm{c}$ & - \\
\hline .V. (\%) & 14,47 & & 12,71 & & 20,29 & & 14,92 & & 22,50 & & 18,02 & \\
\hline
\end{tabular}

${ }^{*} e^{\text {ns }}$ Significativo e não significativo pelo teste $t(p \leq 0,05)$ na comparação das interações de herbicidas. ${ }^{1}$ Médias seguidas de mesma letra minúscula dentro de uma coluna não diferiram significativamente pelo teste de Tukey $(p \leq 0,05)$. Aos tratamentos bentazon+cloransulam e cloransulam foram adicionados adjuvante espalhante adesivo siliconado não iônico a 0,2\%. C.V.= Coeficiente de variação. ${ }^{2}$ Bentazon $\left(720 \mathrm{~g}\right.$ i.a. ha $\left.{ }^{-1}\right)$, Cloransulam - methyl $(17,5 \mathrm{~g}$ i.a. ha-1), Imazethapyr (100 g i.a. ha-1), Cloransulam-methyl + Bentazon $\left(17,5+720 \mathrm{~g}\right.$ i.a. ha $\left.{ }^{-1}\right)$, Imazethapyr + Bentazon $(100+$ 720 g i.a. ha $\left.^{-1}\right)$.

Os principais sintomas de toxicidade foram, predominantemente, clorose aparente, com redução da expansão foliar e rugosidade das folhas. Esses sintomas foram mais acentuados na cultivar IPR Uirapuru. Para a variável fitotoxidez (Tabela 2) houve interação entre cultivares e tratamentos herbicidas. Aos 7 e 14 DAT, todos os tratamentos apresentaram baixa fitotoxidez visível, com exceção de cloransulam isolado. Aos 21 DAT os tratamentos que causaram maior fitotoxidez foram imazethapyr e cloransulam isolados e imazethapyr associado ao bentazon.

Na comparação entre cultivares, aos 14 DAT, o nível de fitotoxidez diferiu significativamente entre as cultivares nos tratamentos bentazon isolado e cloransulam, em que a cultivar IPR Uirapuru apresentou maior nível de fitotoxidez. Em todas as avaliações de fitotoxidez, ambas cultivares apresentaram menores níveis de injúria com o cloransulam associado ao bentazon, em relação a cloransulam isolado, a exceção da cultivar ANfc9, aos 21 DAT, em que o cloransulam isolado ou associado a bentazon não diferiram. 
Tabela 2. Fitotoxidez (\%) visual das cultivares de feijão ANfc9 e IPR Uirapuru em função dos tratamentos avaliadas aos 7, 14 e 21 dias após a aplicação dos tratamentos (DAT). Vacaria, RS, 2017.

Table 2. Crop injury (\%) of the cultivars ANfc9 and IPR Uirapuru in caused by the treatments, evaluated at 7 , 14 and 21 days after treatments (DAT). Vacaria, RS, 2017.

\begin{tabular}{|c|c|c|c|c|c|c|}
\hline & \multicolumn{2}{|c|}{7 DAT } & \multicolumn{2}{|c|}{14 DAT } & \multicolumn{2}{|c|}{21 DAT } \\
\hline Tratamentos & ANfc9 & $\begin{array}{c}\text { IPR } \\
\text { Uirapuru }\end{array}$ & ANfc9 & $\begin{array}{c}\text { IPR } \\
\text { Uirapuru }\end{array}$ & ANfc9 & $\begin{array}{c}\text { IPR } \\
\text { Uirapuru }\end{array}$ \\
\hline Bentazon² & 4 ns $^{1} 1$ & $5 b$ & $0{ }^{*} \mathrm{c}$ & $4 \mathrm{c}$ & 2 ns $b$ & $3 \mathrm{bc}$ \\
\hline Bentazon+cloransulam & $4 \mathrm{~ns} b$ & $5 \mathrm{~b}$ & $3 \mathrm{~ns} b$ & $4 \mathrm{c}$ & 6 ns $b$ & $3 \mathrm{c}$ \\
\hline Bentazon+imazethapyr & 5 ns $a b$ & $3 \mathrm{~b}$ & $4^{\mathrm{ns}} \mathrm{ab}$ & $5 \mathrm{bc}$ & $14^{\text {ns }} a$ & 9 a \\
\hline Cloransulam & $8^{\text {ns }} \mathrm{a}$ & $12 \mathrm{a}$ & $6 * a$ & $10 \mathrm{a}$ & $7^{\mathrm{ns}} \mathrm{ab}$ & $7 \mathrm{ab}$ \\
\hline Imazethapyr & $4^{\mathrm{ns}} \mathrm{b}$ & $6 \mathrm{~b}$ & 6 ns $^{\text {n }}$ & $8 a b$ & $13^{\text {ns }} a$ & $12 \mathrm{a}$ \\
\hline Testemunha Capinada & $0 \mathrm{~ns}^{\mathrm{n}}$ & $0 \mathrm{c}$ & $0{ }^{\text {ns }} \mathrm{C}$ & $0 \mathrm{~d}$ & $0^{\text {ns }} \mathrm{C}$ & $0 \mathrm{~d}$ \\
\hline Testemunha Infestada & $0{ }^{\text {ns }} \mathrm{C}$ & $0 \mathrm{c}$ & $0^{\text {ns }} \mathrm{C}$ & $0 \mathrm{~d}$ & $0 \mathrm{~ns}^{\mathrm{n}} \mathrm{C}$ & $0 \mathrm{~d}$ \\
\hline
\end{tabular}

C.V. (\%)

$\mathrm{e}^{\text {ns }}$ Significativo e não significativo pelo teste $\mathrm{t}(\mathrm{p} \leq 0,05)$ na comparação de cultivares. ${ }^{1}$ Médias seguidas pela mesma letra minúscula na coluna não diferem significativamente entre si pelo teste de Tukey $(p \leq 0,05)$. Aos tratamentos bentazon+cloransulam e cloransulam foram adicionados adjuvante espalhante adesivo siliconado não iônico a $0,2 \%$. C.V.= Coeficiente de variação. ${ }^{2}$ Bentazon (720 g i.a. ha-1 $)$, Cloransulam - methyl (17,5 g i.a. ha- $\left.{ }^{-1}\right)$ Imazethapyr $(100 \mathrm{~g}$ i.a. $\left.\mathrm{ha}^{-1}\right)$, Cloransulam-methyl + Bentazon (17,5 + 720 g i.a. ha- $\left.{ }^{-1}\right)$ Imazethapyr + Bentazon $(100+720$ g i.a. ha-1 $)$.

De modo geral, para ambas cultivares, o bentazon diminuiu os sintomas de fitotoxidez visual provocados pelo cloransulam. Porém, a associação de bentazon com imazethapyr não diminuiu os sintomas de fitotoxidez visual em ambas cultivares. Em um estudo canadense sobre o cloransulam em pósemergência, na mesma dose utilizada neste experimento sobre o feijão, causou altos níveis de injúria visível na cultura (SOLTANI et al. 2010). Diferentes tipos de feijão apresentam diferentes níveis de tolerância ao herbicida, por exemplo, aos 7 e 14 DAT o feijão preto, cultivar Black Knight, apresentou menos sintomas de fitotoxidez que o feijão branco, cultivar OAC Rex, indicando que diferentes cultivares respondem de forma diferente a herbicidas inibidores da enzima ALS aplicado na mesma dose (BAUER et al. 1995a, SOLTANI et al. 2010) corroborando com os resultados obtidos neste ensaio. Neste experimento, cloransulam provocou maiores níveis de injúria que o imazethapyr, isolado. Resultados opostos foram encontrados por VANGESSEL et al. (2000) em que cloransulam isolado causou os menores níveis de injúria no feijão Phaseolus lunatis em relação a imazethapyr.

O efeito protetor do bentazon sobre herbicidas inibidores de ALS foi evidente quando associado ao cloransulam, exceto para cultivar ANfc9 aos 21 DAT. Por outro lado, diferentes herbicidas inibidores de ALS apresentam diferentes níveis de injúria, inclusive sobre o efeito protetor de bentazon quando em associação a estes herbicidas. Todavia, o nível de injúria e a tolerância aos herbicidas inibidores de ALS dependem do herbicida utilizado, conforme reportado por HEKMAT et al. (2008), em que oito diferentes tipos de feijão não apresentaram diferenças significativas quanto à fitotoxidez promovida por imazamox associado ao bentazon, sendo isto o dano diretamente proporcional à dose aplicada em pós-emergência. Em contraste, cloransulam na dose testada neste experimento ou o dobro da dose promoveram o mesmo nível de dano em feijão branco, sendo redução no acúmulo de matéria seca de até $41 \%$ o mais pronunciado (SIKKEMA et al. 2004). Porém o bentazon promove diminuição acentuada nos níveis de injúria foliar causada pelo cloransulam (SOLTANI et al. 2012). A tolerância de diferentes cultivares de feijão a herbicidas inibidores de ALS não está correlacionada a diferenças na taxa de metabolização das moléculas do herbicida pela planta, mas sim devido a diferenças na taxa de absorção e translocação do herbicida pelas folhas, sendo que diferentes cultivares possuem diferentes tipos de cera sob a folha e diferentes espessuras de cutículas foliares. De uma cultivar de feijão mais sensível para uma mais tolerante aos herbicidas inibidores de ALS, a taxa de absorção pode ser de $74 \%$ contra $48 \%$ em 24 horas, e cultivares mais sensíveis podem translocar até 1,3 vezes mais rápido que cultivares tolerantes (BAUER et al. 1995a). Sob o ponto de vista dos herbicidas, o bentazon na cultura do feijão age no fotossistema II, interrompendo o fluxo de elétrons provenientes da hidrólise da água que é direcionado ao fotossistema I, diminuindo a produção de assimilados (fixação de carbono) consequentemente, diminuindo a velocidade de translocação do imazethapyr, que se transloca via floema (BAUER et al. 1995b).

$\mathrm{Na}$ associação de bentazon com imazethapyr, doses crescentes do primeiro fazem os efeitos deletérios do imazethapyr serem minimizados proporcionalmente (BAUER et al. 1995b, SOLTANI et al. 
2008). Os autores relataram que quando o imazethapyr estava associado ao bentazon, não ocorreram mudanças nos teores de clorofila em relação as testemunhas, dessa forma evidenciando o efeito protetor que o bentazon oferece sobre o imazethapyr. $O$ efeito protetor, de fato ocorre devido ao antagonismo entre os dois herbicidas, pois quando o imazethapyr radioativo foi aplicado isolado, houve $50 \%$ mais translocação do que quando o herbicida foi associado ao bentazon.

Na variável altura de inserção de primeiro legume (Tabela 3) houve diferença significativa entre as cultivares em todos os herbicidas, exceto cloransulam isolado. Assim, a cultivar IPR Uirapuru apresentou menores alturas de inserção do primeiro legume em relação a ANfc9. Na cultivar ANfc9, não houve redução na altura de inserção do primeiro legume causada pelos tratamentos. Na cultivar IPR Uirapuru, não houve diferença entre os tratamentos e a testemunha capinada.

Tabela 3. Influência dos tratamentos sobre a altura de inserção de primeiro legume, número de legumes por planta, peso de mil grãos e produtividade de grãos, nas cultivares de feijão Anfc9 e IPR Uirapuru. Vacaria, RS, 2017.

Table 3. Influence of the treatments on yield and yield components, first pod height, number of pods and weight of a thousand grains on the common beans cultivars ANfc9 and IPR Uirapuru. Vacaria, RS, 2017.

\begin{tabular}{|c|c|c|c|c|c|c|c|c|}
\hline \multirow[b]{2}{*}{ Tratamentos } & \multicolumn{2}{|c|}{$\begin{array}{l}\text { Altura de inserção de } \\
\text { primeiro legume }(\mathrm{cm})\end{array}$} & \multicolumn{2}{|c|}{$\begin{array}{c}\text { Número de legumes } \\
\text { por planta }\end{array}$} & \multicolumn{2}{|c|}{ Peso de mil grãos (g) } & \multicolumn{2}{|c|}{ Produtividade $(\mathrm{Kg} / \mathrm{Ha})$} \\
\hline & ANfc9 & $\begin{array}{c}\text { IPR } \\
\text { Uirapuru }\end{array}$ & ANfc9 & $\begin{array}{c}\text { IPR } \\
\text { Uirapuru }\end{array}$ & ANfc9 & $\begin{array}{c}\text { IPR } \\
\text { Uirapuru }\end{array}$ & ANfc9 & $\begin{array}{c}\text { IPR } \\
\text { Uirapuru }\end{array}$ \\
\hline${\text { Bentazon }{ }^{2}}$ & $27 A^{1} a$ & $15 \mathrm{~B}$ ab & $57 \mathrm{~A} \mathrm{a}$ & $61 \mathrm{~A} \mathrm{bc}$ & $208 \mathrm{~A} a$ & $162 \mathrm{~B} \mathrm{~b}$ & $812 \mathrm{~A} \mathrm{C}$ & $198 \mathrm{~B} \mathrm{~d}$ \\
\hline Bent+cloran & $22 \mathrm{~A} a b$ & $16 \mathrm{~B} a b$ & $68 \mathrm{~A} \mathrm{a}$ & $90 \mathrm{~A} \mathrm{abc}$ & $214 \mathrm{~A} \mathrm{a}$ & $163 \mathrm{~B} b$ & $940 \mathrm{~A}$ bc & $582 \mathrm{~B}$ bc \\
\hline Bent+imazet & $19 \mathrm{~A} \mathrm{bc}$ & $12 \mathrm{~B} \mathrm{~b}$ & $73 \mathrm{~B} a$ & $140 \mathrm{~A} \mathrm{a}$ & $214 \mathrm{~A} \mathrm{a}$ & $176 \mathrm{~B} a b$ & $1082 \mathrm{~B} a b c$ & $1337 \mathrm{~A} a$ \\
\hline Cloransulam & $18 \mathrm{~A} \mathrm{bc}$ & $13 \mathrm{~A} a b$ & $68 \mathrm{~A} a$ & $102 \mathrm{~A} a b$ & $208 \mathrm{~A} \mathrm{a}$ & $161 \mathrm{~B} \mathrm{~b}$ & $1161 \mathrm{~A} a b$ & $496 \mathrm{~B} \mathrm{~cd}$ \\
\hline Imazethapyr & $23 \mathrm{~A} a b$ & $11 \mathrm{~B} \mathrm{~b}$ & $55 \mathrm{~B} a$ & $133 \mathrm{~A} \mathrm{a}$ & $217 \mathrm{~A} \mathrm{a}$ & $166 \mathrm{~B} a b$ & $1229 \mathrm{~A} a b$ & $915 B b$ \\
\hline Test. Capin & $14 \mathrm{Ac}$ & $12 \mathrm{Ab}$ & $87 \mathrm{~B} \mathrm{a}$ & $133 \mathrm{~A} \mathrm{a}$ & $222 \mathrm{~A}$ a & $184 \mathrm{~B} \mathrm{a}$ & $1402 \mathrm{~A} a$ & $1323 \mathrm{~A} \mathrm{a}$ \\
\hline Test. Infest & $21 \mathrm{~A} a b$ & $20 \mathrm{~A} \mathrm{a}$ & $45 \mathrm{~A} a$ & $39 \mathrm{Ac}$ & $212 \mathrm{~A} \mathrm{a}$ & $183 \mathrm{~B} \mathrm{a}$ & $372 \mathrm{Ad}$ & $182 \mathrm{Ad}$ \\
\hline
\end{tabular}

${ }^{1}$ Médias seguidas pela mesma letra maiúscula na linha e minúscula na coluna não diferem significativamente entre si pelo teste de Tukey $(p \leq 0,05)$. Aos tratamentos bentazon+cloransulam e cloransulam foram adicionados o adjuvante espalhante adesivo siliconado não iônico a 0,2\%. C.V.= Coeficiente de variação. ${ }^{2}$ Bentazon $\left(720 \mathrm{~g}\right.$ i.a. ha $\left.{ }^{-1}\right)$, Cloransulam - methyl (17,5 g i.a. ha-1 $)$, Imazethapyr (100 g i.a. ha-1), Cloransulam-methyl + Bentazon (17,5 + 720 g i.a. ha $\left.^{-1}\right)$, Imazethapyr + Bentazon $\left(100+720\right.$ g i.a. ha $\left.{ }^{-1}\right)$.

$\mathrm{Na}$ variável número de legumes por plantas e peso de mil grãos (Tabela 3), houve diferença significativa entre as cultivares, porém isto provavelmente é uma característica das cultivares testadas, pois na testemunha capinada as cultivares também diferiram. Para a variável número de legumes por planta, na cultivar ANfc9, não houve diferença significativa entre os tratamentos. Já na cultivar IPR Uirapuru, os únicos tratamentos que causaram redução na produção do número de legumes foi o bentazon que se equivaleu à testemunha infestada. Na variável peso de mil grãos (PMG) não houve diferença entre tratamentos para a cultivar ANfc9, porém em IPR Uirapuru o PMG foi significativamente menor em relação as testemunhas nos tratamentos contendo cloransulam associado ao bentazon e isolado e bentazon isolado. É importante observar que todos os tratamentos com herbicidas causaram redução do peso de mil grãos na cultivar IPR Uirapuru. Estes resultados diferem de BAUER et al. (1995a) nos quais reportaram que imazethapyr na metade da dose testada neste experimento causou fitotoxidez elevada e consequentemente necrose do meristema apical levando a maior emissão de brotações laterais e assim maior número de legumes nas plantas.

$\mathrm{Na}$ variável produtividade (Tabela 3), houve diferença significativa nas cultivares causadas pelos tratamentos. Houve redução de produtividade na cultivar IPR Uirapuru em relação a ANfc9 causada pelos herbicidas bentazon isolado e associado ao cloransulam, os quais diferiram da testemunha capinada. Por outro lado, o tratamento bentazon associado ao imazethapyr causou redução significativa da cultivar ANfc9 em relação a IPR Uirapuru. Para a cultivar ANfc9, os tratamentos de bentazon isolado e associado ao 
cloransulam causaram redução de produtividade, enquanto que na cultivar IPR Uirapuru somente bentazon associado ao imazethapyr proporcionou maior produtividade e bentazon isolado e cloransulam se equivaleram a testemunha infestada.

A menor produtividade com os tratamentos contendo cloransulam ocorreram devido à infestação de milhã que ocasionou redução na produtividade, principalmente da cultivar IPR Uirapuru (Tabela 3). Na cultivar IPR Uirapuru, para a variável produtividade ficou evidente o efeito protetor do bentazon sobre 0 imazethapyr pois a produtividade foi superior a produtividade dos herbicidas de forma isolada; também, esse tratamento apresentou equivalência a testemunha capinada. O imazethapyr isolado é capaz de reduzir em até 8 dias o ciclo produtivo do feijão, pela diminuição dos teores de clorofila das folhas devido às lesões e eventual necrose que o herbicida promove, o que diminui o acúmulo de fotoassimilados armazenados nas sementes levando à queda de produtividade. Entretanto, quando em associação com o bentazon, esses efeitos deletérios não ocorrem e a produtividade não é afetada, podendo a planta produzir pelo mesmo período que a testemunha não tratada (BAUER et al. 1995b). Entretanto, como a tolerância a herbicidas inibidores de ALS dependem de fatores genéticos quantitativos (BAUER et al. 1995a), outros tipos ou cultivares de feijão podem apresentar menor susceptibilidade a herbicidas inibidores de ALS, que podem apresentar produtividade ao mesmo nível de plantas não tratadas mesmo em dose 1,5 vezes maior que a utilizada neste experimento (SOLTANI et al. 2008).

\section{CONCLUSÃO}

As duas cultivares de feijão avaliadas neste experimento possuem níveis diferentes de tolerância aos herbicidas cloransulam e imazethapyr. A associação dos herbicidas cloransulam ou imazethapyr com o bentazon não causou antagonismo e redução no controle de picão preto e losna. $O$ efeito do imazethapyr sobre o controle de milhã não foi afetado pelo bentazon. A cultivar Anfc9 possui maior tolerância ao cloransulam, comparativamente a cultivar IPR Uirapuru. Assim, o herbicida cloransulam isolado ou associado ao bentazon, na dose testada, tem potencial de uso na cultura do feijão.

\section{REFERÊNCIAS}

AGROFIT. 2017. Consulta de produtos formulados. Brasília: ministério da agricultura. Disponível em: <http://agrofit.agricultura.gov.br/agrofit_cons/principal_agrofit_cons>. Acesso em 26 fev. 2017.

CONAB. 2016. Companhia Nacional de Abastecimento. Acompanhamento da safra brasileira de grãos. Brasília: Conab. Disponível em: <http://www.conab.gov.br/olalacms/uploads/arquivos/16_01_12_09_00_46_

boletim_graos_janeiro_2016.pdf>. Acesso em: 26 fev. 2017.

BAUER TA et al. 1995a. Pinto bean (Phaseolus vulgaris) varietal tolerance to imazethapyr. Weed Science 43: 417-424.

BAUER TA et al. 1995b. 'Olathe' pinto bean (Phaseolus vulgaris) response to postemergence imazethapyr and bentazon. Weed Science 43: 276-282.

BAUER TA et al. 1995c. Response of selected weed species to postemergence imazethapyr and bentazon. Weed Technology 9: 236-242.

CANTWELL JR et al. 1989. Imazethapyr for weed control in soybean (Glycine max). Weed Technology 3: 596-601.

COLBY SR. 1967. Calculating synergistic and antagonistic responses of herbicide combinations. Weeds 15: 20-22.

CONSTANTIN J. 2011. Métodos de manejo. In: OLIVEIRA Jr. et al. (Eds.) Biologia e Manejo de Plantas Daninhas. Curitiba: Omnipax. p. 67-78.

HEKMAT $S$ et al. 2008. Effect of imazamox plus bentazon on dry bean (Phaseolus vulgaris L.). Crop Protection 27: 1491-1494.

KOZLOWSKI LA et al. 2002. Período crítico de interferência das plantas daninhas na cultura do feijoeiro-comum em sistema de semeadura direta. Planta Daninha 20: 213-220.

SIKKEMA PH et al. 2004. Tolerance of white bean to postemergence broadleaf herbicides. Weed Technology 18: 893901.

SOLTANI N et al. 2008. Response of pinto and small red Mexican bean to postemergence herbicides. Weed Technology 22: 195-199.

SOLTANI N et al. 2010. Tolerance of black, cranberry, kidney, and white bean to cloransulam-methyl. Weed Biology and Management 10: 33-39.

SOLTANI N et al. 2012. Safening effect of bentazon on cloransulam-methyl and halosulfuron-methyl in dry bean. Agricultural Sciences 3: 368-374.

SOLTANI N et al. 2013. Weed management in white beans with postemergence herbicide tankmixes. Canadian Journal of Plant Sciences 93: 669-674.

VANGESSEL MJ et al. 2000. Herbicides for potential use in lima bean (Phaseolus lunatus) production. Weed Technology 14: 279-286. 